\title{
MicroRNA Therapeutics for Cardiac Disease
}

\author{
Yaoping Tang, Ndah A Poteh, Angela Chiang and II-man Kim* \\ Vascular Biology Center\& Department of Biochemistry and Molecular Biology, Medical College of Georgia, Georgia Regents University, Augusta, GA, USA
}

Cardiac disease is a major health concern among the elderly and obese populations. Being the number one cause of death in America, it is rapidly becoming a popular topic for researchers nowadays. MicroRNAs (miRs) are small, non-coding RNA molecules that range from 19 to 25 nucleotides in length and regulate gene expression mainly via binding to mRNAs and causing the repression of their expression $[1,2]$. They serve as intracellular mediators that are necessary for the heart to function properly. It has been shown that the causes of some cardiovascular diseases can be linked to aberrant expression of MiRs [3]. Therefore, these tiny molecules are of immense importance and have become a target for drug therapy in heart disease.

\section{MicroRNA biogenesis}

MiR genes can be found in a genome either as individual transcriptional units or as clusters of genes [4-7]. MiR is transcribed by RNA polymerase II to yield a long transcript referred to as primary miR. This transcript is then processed by an enzyme called Drosha, to yield a shorter hairpin-shaped/double-stranded RNA referred to as premature $\mathrm{miR}$. The premature $\mathrm{miR}$ then leaves the nucleus and goes into the cytoplasm where it is further processed by a protein called Dicer into mature miR [1,2]. This mature miR binds to target mRNAs, causing the repression of gene expression.

\section{MicroRNAs in the heart}

MiRs regulate cardiac cell growth, differentiation and survival. Among thousands of miRs that have been discovered, 18 are very highly expressed in the heart, accounting for about $90 \%$ of total expression. During the progression of heart disease, the expression of nearly all $18 \mathrm{miRs}$ is dysregulated. Several of them belong to families, including let-7, miR-30 and miR-29 families [3]. MiR-21 is the most upregulated $\mathrm{miR}$ in cardiovascular diseases and is highly expressed in the different types of cardiac cells [2]. It is upregulated during the ballooning of arteries as well as in cardiac hypertrophy. This is the same for other miRs; their upregulation leads to cardiovascular diseases [3]. Given the fact that miRs regulate many pathophysiological processes, future drug therapy, which uses oligonucleotides that are chemically modified to either target certain miRs or affect their binding to a particular mRNA target, is warranted.

\section{The role of microRNAs in cardiac disease}

MiRs serve as important and powerful mediators in cardiac disease and cardiac system. Many miRs have been discovered to be involved in cardiomyocyte differentiation both in vitro and in vivo [8,9]. MiR-133a is one of them, which affects both cardiac development and pathological hypertrophy [10]. After Kwon et al. [11] reported a miR that can affect cardiac cell proliferation; increasing numbers of studies have shown abnormal expression of certain miRs in relation to cardiac disease. MiR-1, miR-21, miR-24, miR-29, miR-33,miR-92a, miR-126, miR-133, miR-199a, miR-208, miR-208a, miR-195, miR-320, and miR-499 have been shown to be involved in the pathogenesis of acute myocardial infarction, heart failure, cardiac injury, arrhythmias, fibrosis, pressure overload-induced remodeling, metabolic disorders and other cardiac diseases [12,13]. Although miRs can exacerbate cardiac disease, their expression can also prevent the disease [14]. Research has shown that
miRs can simultaneously regulate multiple targets and that many miRs can bind to the same target, suggesting that $\mathrm{miR}$ regulation in cardiac system and cardiac disease is complex. Despite all the progress, we still need more research, particularly in large animals and clinical studies to further explore and confirm the role of miRs in cardiac disease.

Scientists also pay attention to the risk factors of cardiac disease that might be caused by miRs. For example, Wang et al. summarized that many miRs are involved in the pathogenesis of blood pressure, cholesterol, obesity and diabetes [13], indicating that miRs play an important role in modifying the risk factors of cardiac disease. Research also shows that miRs can modulate numerous ion channels involved in cardiac rhythm $[14,15]$. This line of research along with the recent identification of miRs in the circulation bring up the possibility of reducing mortality of cardiac disease via utilizing miRs as potential biomarkers for early diagnosis and early treatment.

\section{MicroRNA-based therapies in cardiac disease}

A wide variety of recent studies suggest that miRs act as intracellular and intercellular mediators in cardiac disease [16]. Based on these mechanisms, miRs have been considered as potential prognostic biomarkers, resulting in novel pharmacological therapeutic strategies $[17,18]$. Krutzfeldt et al. also showed that antagomirs were able to knock down miR expression in cardiac tissue [19]. Thum et al. showed that anantagomir against fibroblast-enriched miR-21 prevented cardiac fibrosis [18]. Other studies also successfully confirmed that miR inhibitors conferred cardiovascular protective functions [20]. Thus, both antagomirs and miR inhibitors can effectively target miRs in vitro and in vivo. Recently, Wang et al. used miR erasers and sponges as additional tools to decrease $\mathrm{miR}$ expression [21]. These anti-miR strategies are employed to suppress the expression of target miRs that are upregulated in cardiac disease. When miRs are down regulated in cardiac disease, miR mimic technology, which uses small, chemically modified double-stranded RNA molecules that are designed to mimic endogenous mature miRs, can be utilized to recover the expression of target miRs $[20,22]$. Therefore, both positive and negative regulation in the expression of miRs can be used as a therapeutic means.

A recent study compared intravenous, intraperitoneal and subcutaneous delivery routes of anti-miR-208a in mice. All these administration methods showed similar results that the inhibition of miR-208a improved cardiac function during heart failure [23]. Other studies also compared the effects of various doses of cholesterol-based

*Corresponding author: Il-man Kim, Assistant Professor, Vascular Biology Center \& Department of Biochemistry and Molecular Biology, Medical College of Georgia, Georgia Regents University, 1459 Laney Walker Blvd,CB-3717, Augusta GA 30912, USA, Tel: 706-721-9414; Fax: 706-721-9799; E-mail: ILKIM@gru.edu

Received June 29, 2013; Accepted July 01, 2013; Published July 05, 2013

Citation: Tang Y, Poteh NA, Chiang A, Kim I (2013) MicroRNA Therapeutics for Cardiac Disease. Cardiol Pharmacol 2: e113. doi:10.4172/2329-6607.1000e113

Copyright: (c) 2013 Tang Y, et al. This is an open-access article distributed under the terms of the Creative Commons Attribution License, which permits unrestricted use, distribution, and reproduction in any medium, provided the original author and source are credited. 
antagomirs on cell-type specific uptake and silencing of miR-24 [24]. The results indicated that the optimal doses are dependent on individual miRs and their abundance in the target organ. Nevertheless, the costs for miR-modulating chemistry have been reduced significantly so that large animal and clinical studies are now possible these days.

Despite great advances and contributions of miR-targeted therapies to cardiac disease, there are still a few drawbacks. For example, if we modulate a diverse set of target mRNAs by manipulating the expression of a specific miR, both beneficial and pathological processes will be affected. Our major concerns are that a specific cardiac disease condition might be modulated by multiple miRs, and that one gene can be regulated by multiple miRs. Henceforth, the challenge is to understand specific biological actions of miRs. Although current miR-based therapy in experimental models has produced favorable outcomes, there are pending issues that need to be resolved prior to the consideration of miR-based gene therapy in the clinic. These include the need for definitive mRNA target validation, our incomplete understanding of rate-limiting cellular components that impact the efficiency of this post transcriptional gene-silencing phenomenon, the possibility for nonspecific immune activation, and the lack of a defined, optimal mode of delivery.

\section{Modulating upstream signaling pathways to regulate miR biogenesis as a potential therapy in cardiac disease}

Recent research has shown that some upstream signaling pathways can regulate miR biogenesis, thereby modulating downstream targets in cardiac disease. It was demonstrated that IGF-1 pathway controls miR-1 expression in cardiac muscle [25]. MiR-29 is down regulated by TGF- $\beta 1$ in the fibrotic tissues after myocardial infarction [26]. TGF $\beta$, Wnt and Notch signaling pathways can also modulate the biogenesis of a subset of miRs in cardiac disease [27]. Indeed, signaling pathways are ideal targets for regulating miR activity because of the sharp dosesensitive nature of their effects. Because cell signaling pathways have been shown to modulate the activities of some components of the miR biogenesis, it is possible to achieve optimal miR expression patterns by regulating the upstream signaling pathways. Therefore, one can develop a novel drug for cardiac disease, where upstream signaling pathways modulate causative $\mathrm{miR}$ expression. Future studies are needed to identify additional pathways affecting these important signal transduction networks and to elucidate the underlying mechanisms of how different signaling pathways regulate the specific miRs.

\section{Conclusion remarks}

Despite the many novel findings that have been identified in the miRbased regulation of gene expression in the cardiovascular system, many gaps still exist. For example, the detailed mechanism of combinatorial interactions between multiple miRs and coordinated target mRNAs, which is likely to play an important role in the regulation of genes and physiological pathways, is still not fully understood. Furthermore, few large animal and human studies have been done. To completely translate exciting basic research outcomes into clinical therapeutic options, further collaborative research has to be pursued. Due to the fact that miR-based therapies using chemically modified oligonucleotides have several disadvantages, we anticipate that modulating the upstream signaling pathways to regulate miR biogenesis will represent novel therapeutic targets for the future.

\section{Acknowledgements}

We would like to thank editorial office for giving us an opportunity to write this editorial. We apologize to those colleagues whose references were omitted from this discussion; due to space restrictions and our narrow focus, we were unable to include all articles on this interesting and diverse subject matter. We would like to express our gratitude to the members of the Kim lab for their contributions. This work was supported by Georgia Regents University (GRU) Diabetes and Obesity Discovery Institute Scholar Program to NAP and AC, American Heart Association Grant-in-Aid (12GRNT12100048) and GRU Departmental Start-Up Fund to IK.

\section{References}

1. Shukla GC, Singh J, Barik S (2011) MicroRNAs: processing, maturation, target recognition and regulatory functions. Mol Cell Pharmacol 3: 83-92.

2. Cheng Y, Zhang C (2010) MicroRNA-21 in cardiovascular disease. J Cardiovasc Transl Res 3: 251-255

3. Small EM, Frost RJ, Olson EN (2010) MicroRNAs add a new dimension to cardiovascular disease. Circulation 121: 1022-1032.

4. Lagos-Quintana M, Rauhut R, Lendeckel W, Tuschl T (2001) Identification of novel genes coding for small expressed RNAs. Science 294: 853-858.

5. Lau NC, Lim LP, Weinstein EG, Bartel DP (2001) An abundant class of tiny RNAs with probable regulatory roles in Caenorhabditis elegans. Science 294: 858-862.

6. Lee Y, Jeon K, Lee JT, Kim S, Kim VN (2002) MicroRNA maturation: stepwise processing and subcellular localization. EMBO J 21: 4663-4670.

7. Reinhart BJ, Slack FJ, Basson M, Pasquinelli AE, Bettinger JC, et al. (2000) The 21-nucleotide let-7 RNA regulates developmental timing in Caenorhabditis elegans. Nature 403: 901-906.

8. Wilson KD, Hu S, Venkatasubrahmanyam S, Fu JD, Sun N, et al. (2010) Dynamic microRNA expression programs during cardiac differentiation of human embryonic stem cells: role for miR-499. Circ Cardiovasc Genet 3: 426435.

9. Zhao Y, Ransom JF, LiA, Vedantham V, von Drehle M, et al. (2007) Dysregulation of cardiogenesis, cardiac conduction, and cell cycle in mice lacking miRNA-1-2. Cell 129: 303-317.

10. Liu N, Bezprozvannaya S, Williams AH, Qi X, Richardson JA, et al. (2008) MicroRNA-133a regulates cardiomyocyte proliferation and suppresses smooth muscle gene expression in the heart. Genes Dev 22: 3242-3254.

11. Kwon C, Han Z, Olson EN, Srivastava D (2005) MicroRNA1 influences cardiac differentiation in Drosophila and regulates Notch signaling. Proc Natl Acad Sci U S A 102: 18986-18991.

12. JanosiA (2005) Epidemiology and prevention of cardiovascular diseases. Orv Hetil 146: 683-688.

13. Wang Y, Teoh JP, Park KM, Tang Y, Krothapalli S, et al. (2013) MicroRNAs as diagnostic, prognostic and predictive biomarkers of cardiac disease. Recent Patents on Biomarkers 3: 81-97.

14. Small EM, Olson EN (2011) Pervasive roles of microRNAs in cardiovascular biology. Nature 469: 336-342.

15. Yang B, Lin H, Xiao J, Lu Y, Luo X, et al. (2007) The muscle-specific microRNA miR-1 regulates cardiac arrhythmogenic potential by targeting GJA1 and KCNJ2. Nature Med 13: 486-491.

16. da Costa Martins PA, Bourajjaj M, Gladka M, Kortland M, van Oort RJ, et al (2008) Conditional dicer gene deletion in the postnatal myocardium provokes spontaneous cardiac remodeling. Circulation 118: 1567-1576.

17. Widera C, Gupta SK, Lorenzen JM, Bang C, Bauersachs J, et al. (2011) Diagnostic and prognostic impact of six circulating microRNAs in acute coronary syndrome. J Mol Cell Cardiol 51: 872-875.

18. Thum T, Gross C, Fiedler J, Fischer T, Kissler S, et al. (2008) MicroRNA-21 contributes to myocardial disease by stimulating MAP kinase signalling in fibroblasts. Nature 456: 980-984.

19. Krutzfeldt J, Rajewsky N, Braich R, Rajeev KG, Tuschl T, et al.(2005) Silencing of microRNAs in vivo with 'antagomirs'. Nature 438: 685-689.

20. Pan ZW, Lu YJ, Yang BF (2010) MicroRNAs: a novel class of potentia therapeutic targets for cardiovascular diseases. Acta Pharmacol Sin 31: 1-9.

21. Wang $Z$ (2011) The concept of multiple-target anti-miRNA antisense oligonucleotide technology. Methods Mol Biol 676: 51-57

22. Thum T (2012) MicroRNA therapeutics in cardiovascular medicine. EMBO Mol Med 4: 3-14. 
23. Montgomery RL, Hullinger TG, Semus HM, Dickinson BA, Seto AG, et al. (2011) Therapeutic inhibition of miR-208a improves cardiac function and survival during heart failure. Circulation 124: 1537-1547.

24. Fiedler J, Jazbutyte V, Kirchmaier BC, Gupta SK, Lorenzen J, et al. (2011) MicroRNA-24 regulates vascularity after myocardial infarction. Circulation 124: 720-730

25. Elia L, Contu R, Quintavalle M, Varrone F, Chimenti C, et al. (2009) Reciprocal regulation of microRNA-1 and insulin-like growth factor-1 signal transduction cascade in cardiac and skeletal muscle in physiological and pathological conditions. Circulation 120: 2377-2385.

26. van Rooij E, Sutherland LB, Thatcher JE, DiMaio JM, Naseem RH, et al. (2008) Dysregulation of microRNAs after myocardial infarction reveals a role of miR29 in cardiac fibrosis. Proc Natl Acad Sci U S A 105: 13027-13032.

27. Inui M, Martello G, Piccolo S (2010) MicroRNA control of signal transduction Nat Rev Mol Cell Biol 11: 252-263. 